\title{
MD2 blockage prevents the migration and invasion of hepatocellular carcinoma cells via inhibition of the EGFR signaling pathway
}

\author{
Yajun $\mathrm{Qi}^{1,2 \#}$, Qilu Fang ${ }^{1,2 \#}$, Qinglin $\mathrm{Li}^{2,3}$, Haiying Ding ${ }^{1}$, Qi Shu ${ }^{1,2}$, Yan $\mathrm{Hu}^{1,2}$, Wenxiu Xin ${ }^{1,2,3}$, Luo Fang ${ }^{1,2}$ \\ ${ }^{1}$ Department of Pharmacy, The Cancer Hospital of the University of Chinese Academy of Sciences (Zhejiang Cancer Hospital), Hangzhou, China; \\ ${ }^{2}$ Institute of Basic Medicine and Cancer (IBMC), Chinese Academy of Sciences, Hangzhou, China; ${ }^{3}$ Department of Comprehensive Medical \\ Oncology, Key Laboratory of Head and Neck Translational Research of Zhejiang Province, Zhejiang Cancer Hospital, Hangzhou, China \\ Contributions: (I) Conception and design: W Xin, L Fang; (II) Administrative support: H Ding; (III) Provision of study materials or patients: All \\ authors; (IV) Collection and assembly of data: All authors; (V) Data analysis and interpretation: Y Qi, Q Fang; (VI) Manuscript writing: All authors; \\ (VII) Final approval of manuscript: All authors. \\ "These authors contributed equally to this work. \\ Correspondence to: Wenxiu Xin; Luo Fang. Department of Pharmacy, The Cancer Hospital of the University of Chinese Academy of Sciences (Zhejiang \\ Cancer Hospital), No. 1, Banshan Road, Hangzhou 310022, China. Email: xinwx@zjcc.org.cn; fangluo@zjcc.org.cn.
}

Background: The toll-like receptor (TLR) is an emerging signaling pathway in tumor invasion and metastasis. The activation of TLRs requires specific accessory proteins, such as the small secreted glycoprotein myeloid differentiation protein 2 (MD2), which contributes to ligand responsiveness. However, the role of MD2 in tumorigenesis and metastasis has rarely been reported. This study aimed to investigate the effects and underlying mechanisms of MD2 on the proliferation, migration, and invasion of hepatocellular carcinoma (HCC).

Methods: Cell counting kit 8 (CCK8), cell colony formation, wound healing, and transwell assays were conducted to determine cell viability, proliferation, migration, and invasion, respectively. Quantitative realtime PCR (qRT-PCR) was performed to assess the expression of MD2 in HCC cell lines and human normal liver cell lines as well as the silencing efficiency of MD2 blockage. Western blot and qRT-PCR assays were performed to detect the protein and mRNA expression levels of epithelial mesenchymal transformation (EMT) markers and epidermal growth factor receptor (EGFR) signaling molecules.

Results: MD2 was highly expressed in HCC tissues and cell lines. High expression of MD2 was associated with poor prognosis of HCC patients. In addition, MD2 silencing slightly inhibited the proliferation of HepG2 and HCCLM3, and significantly suppressed cell migration and invasion. Furthermore, MD2 blockage could distinctly prevent the EMT process by increasing the protein and mRNA levels of E-cadherin and Occludin, and decreasing the levels of Vimentin, N-cadherin, and Snail. Finally, the phosphorylation level of EGFR as well as its downstream molecular Src, Akt, I- $\kappa \mathrm{B} \alpha$, and p65 were downregulated in HCC cells with MD2 silencing.

Conclusions: Our findings suggest that high expression of MD2 may affect the EMT, migration, and invasion via modulation of the EGFR pathway in HCC cells.

Keywords: Hepatocellular carcinoma (HCC); myeloid differentiation protein 2 (MD2); epidermal growth factor receptor (EGFR); migration; invasion

Submitted Jun 02, 2021. Accepted for publication Jul 29, 2021.

doi: 10.21037/jgo-21-362

View this article at: https://dx.doi.org/10.21037/jgo-21-362

^ ORCID: 0000-0001-5491-4930. 


\section{Introduction}

Hepatocellular carcinoma (HCC) is the fourth most common cause of cancer-related mortality worldwide, and accounts for more than $80 \%$ of all liver cancer cases (1). More than $70 \%$ of HCC patients experienced tumor progression 1 year after diagnosis due to the extensive invasiveness of HCC $(2,3)$, and the 5 -year survival rate of advanced HCC is very poor (below $15 \%)(4,5)$. Thus, exploring the potential therapeutic targets for HCC is crucial.

Myeloid differentiation protein 2 (MD2), also known as lymphocyte antigen 96 (LY96), is a kind of small secreted protein (6). As the co-receptor for toll-like receptor (TLR) 4 and the main lipopolysaccharide (LPS)-binding module, MD2 is requisite for the localization of TLR4 on the cell surface and subsequently confers responsiveness to LPS $(7,8)$. MD2 mediates the LPS-induced inflammatory and immune responses by forming a heterotetrametric complex with TLR4 $(9,10)$. The TLR4 signaling pathway is an emerging pathway related to tumor cell invasion and migration (11-13). TLR4 signaling has been shown to have oncogenic effects both in vitro and in vivo in various cancer types, including colon (14), breast (15), prostate (16), skin (17), ovarian (18), cervical (19), and esophageal (20) cancers, as well as HCC (21).

Considering the essential role of MD2 in TLR4 signaling activation, targeting MD2 may be a potential pharmacological strategy for cancer treatment. However, the effect of MD2 on tumor cell invasion and migration has been rarely studied, and no studies addressing drugs targeting MD2 for cancer treatment specifically are currently available. Data from the TNMplot database (https://tnmplot.com) indicates that MD2 is overexpressed in human HCC tissues compared with normal liver tissues. Furthermore, the data of a 5 -year analysis from the KaplanMeier plotter database (https://kmplot.com/analysis/index. php) shows that high expression of MD2 in tumor tissues is associated with poor prognosis of HCC. Although the role of MD2 in HCC is still unclear, these data point to the intriguing possibility that MD2 might play a role in HCC.

This study aims to evaluate the precise mechanisms of MD2 on the invasion and migration of HCC. Our findings indicate that MD2 is involved in the migration and invasion of HCC cells, and its mechanism may be related to the regulation of the epidermal growth factor receptor (EGFR) signaling pathway. We present the following article in accordance with the MDAR reporting checklist (available at https://dx.doi.org/10.21037/jgo-21-362).

\section{Methods}

\section{Cell culture}

Human hepatoma carcinoma cell lines (Hep3B, HepG2, HCCLM3, and Huh7) and normal hepatocyte (HL7702) were obtained from the National Collection of Authenticated Cell Cultures (NSTI, Shanghai, China), and cultured in RPMI-1640 (Gibco, Grand Island, USA) supplemented with $10 \%$ fetal bovine serum (Gibco) and 1\% penicillin-streptomycin (Invitrogen, Beijing, China) at $37^{\circ} \mathrm{C}$ in a $5 \% \mathrm{CO}_{2}$ cell incubator.

\section{siRNA transfections}

Cells were transfected with $4-5 \mathrm{ng} / \mathrm{mL}$ siRNA against MD2 (GenePharma, Shanghai, China) and a negative control (GenePharma, Shanghai, China) using Lipofectamine 3000 (Thermo Fisher Scientific, USA) for $24 \mathrm{~h}$. Cells were then washed and cultured in fresh complete medium and the silencing efficiencies were detected by western blot and quantitative real-time PCR (qRT-PCR).

\section{qRT-PCR analysis}

RNA was isolated from each group as described in individual experiments using TRIzol (Invitrogen, cat. no. 15596026). cDNA was synthesized with $2-5 \mu \mathrm{g}$ of RNA using a PrimeScript RT reagent kit (TaKaRa, Tokyo, Japan). Quantitative PCR was carried out using a SYBR ${ }^{\circledR}$ Premix Ex Taq ${ }^{\mathrm{TM}}$ II kit (TaKaRa, cat. no. RR820A). The mRNA levels of MD2 (forward primer: 5'-TGCCAGAAGACCGTGTACC-3', reverse primer: 5'-TTTGCAGTAGAACTCCCCGTG-3'), Vimentin (forward primer: 5'-GACGCCATCAACACCGAGTT-3', reverse primer: 5'-CTTTGTCGTTGGTTAGCTGGT-3'), N-cadherin (forward primer: 5'-AGCTCCATTCCGACTTAGACA-3'; reverse primer: 5'-CAGCCTGAGCACGAAGAGTG-3'), Snail (forward primer: 5'- TGTGACAAGGAATATGTGAGCC-3', reverse primer: 5'-TGAGCCCTCAGATTTGACCTG-3'), E-cadherin (forward primer: 5'-ATTTTTCCCTCGACACCCGAT-3', reverse primer: 5'-TCCCAGGCGTAGACCAAGA-3'), Occludin (forward primer: 5'-ACAAGCGGTTTTATCCAGAGTC-3', reverse primer: 5'- GTCATCCACAGGCGAAGTTAAT-3'), and GAPDH (forward primer: 5'-TGTGGGCATCAATGGATTTGG-3'; reverse primer: 5'-ACACCATGTAT TCCGGGTCAAT-3') were quantified using the LightCycle* 480 II (Roche Diagnostic, QC, Canada). 


\section{Western blot analysis}

Lysates of total cells were extracted as described in individual experiments using lysis buffer with protease inhibitor, and the total protein concentration was analyzed using a BCA Protein Assay Kit (Beyotime, \#P0012, Shanghai, China) according to the manufacturer's protocol. Samples were separated on $8-12 \%$ SDS-PAGE gels and transferred to PVDF membranes (Millipore, IPVH00010, USA), and subsequently blocked with $5 \%$ non-fat dry milk for $1.5 \mathrm{~h}$ at room temperature and incubated overnight at $4{ }^{\circ} \mathrm{C}$ with appropriate primary antibodies: anti-MD2 (1:3,000, \#ab24182, Abcam), E-cadherin (1:3,000, \#20874-1-AP, Proteintech), Occludin (1:3,000, \#27260-1-AP, Proteintech), Vimentin (1:3,000, \#10366-1-AP, Proteintech), Snail (1:3,000, \#13099-1-AP, Proteintech), p-EGFR (F-3) (1:3,000, \#sc377547, Santa Cruz), EGFR (1:3,000, \#sc373746, Santa Cruz), p-Src (Y419) (1:1,000, \#6943S, Huabio), Src (1:3,000, \#2109, Cell Signaling), p-ІкB- $\alpha$ (B-9) (1:1,000, \#sc8404, Santa Cruz), p-p65 (1:3,000, \#3033, Cell Signaling), p-AKT (Ser473) (1:3,000, \#4060, Cell Signaling), AKT (1:3,000, \#4685, Cell Signaling), and GAPDH (1:3,000, \#6004-1-Ig, Proteintech). Horseradish peroxidase-conjugated goat anti-rabbit or anti-mouse antibodies were used as secondary antibodies. Protein signals were visualized using a BeyoECL Plus kit (Beyotime, \#P0018, Shanghai, China) and quantified by ChemiDoc XRS+ software (Bio-Rad, USA).

\section{Wound bealing assay}

Cells were seeded and transfected with siRNA against MD2 and a negative control. Cells were allowed to reach $70-80 \%$ confluence, and were then starved for $24 \mathrm{~h}$. The cells were subsequently wounded with a sterile plastic tip and cultured in serum-free medium. Cell migration was monitored for 24 h using microscopy (Nikon, Tokyo, Japan).

\section{Transwell assay}

Cells were transfected with siRNA against MD2 and a negative control and plated on top of a thick layer of Matrigel in transwell chambers (BD Biosciences, USA). After culturing for 18 hours, non-invasive cells on the upper surface of filters were removed completely. Invasive cells that adhered to the lower surface of the filter were rinsed with phosphate buffered saline (PBS), fixed with methanol, stained with $0.05 \%$ crystal violet, and counted.

\section{Cell viability assay}

Cell counting kit 8 (CCK8) assay was used to determine cell viability. Cells $\left(5 \times 10^{3}\right.$ cells/well $)$ were transfected with siRNA against MD2 and a negative control, laid out in 96-well plates, and incubated for 24, 48, and $72 \mathrm{~h}$. Next, the cell medium was removed and CCK8 reagent was added (diluted with the medium at $1: 10$ ), and incubated at $37^{\circ} \mathrm{C}$ for $30 \mathrm{~min}$. The absorbance was measured at $595 \mathrm{~nm}$ using an enzyme-linked immunosorbent assay (ELISA) microplate reader.

\section{Colony formation assay}

Cells $\left(5 \times 10^{4}\right.$ cells/well) were transfected with siRNA against MD2 or a negative control, and seeded in six-well plates for 5 days. During this period, the medium was renewed every 3 days. Colonies were fixed with $4 \%$ paraformaldehyde for $15 \mathrm{~min}$ and stained with $0.05 \%$ crystal violet at room temperature for $15 \mathrm{~min}$. Images were obtained using a Nikon camera (Nikon, Tokyo, Japan).

\section{Statistical analysis}

All experiments were performed at least in triplicate. Data were analysed using GraphPad Prism Version 9.0.0 (GraphPad Software Inc., La Jolla, CA, USA). Values were presented as means \pm standard deviation (SD). Statistical significance was evaluated using one-way analysis of variance (ANOVA) followed by Tukey's test. A P value of $<0.05$ was considered to be statistically significant.

\section{Results}

\section{MD2 was highly expressed in HCC and was associated with poor prognosis}

To investigate the relationship between MD2 and HCC, we first analyzed the expression of MD2 in hepatoma carcinoma using the TNMplot database, and found that the MD2 gene (LY96) was significantly higher in HCC tumor tissues than in normal liver tissues (Figure 1A). Next, we detected the mRNA level of MD2 in several HCC cell lines, and obtained the same results as above; that is, that $M D 2$ was higher in HCC cell lines (Hep3B, HepG2, HCCLM3, and Huh7) than in normal liver cell line (HL7702) (Figure 1B). Furthermore, we analyzed the prognosis via the Kaplan-Meier plotter database, and found that the 5-year survival rate of patients with lower MD2 expression was higher than those with 

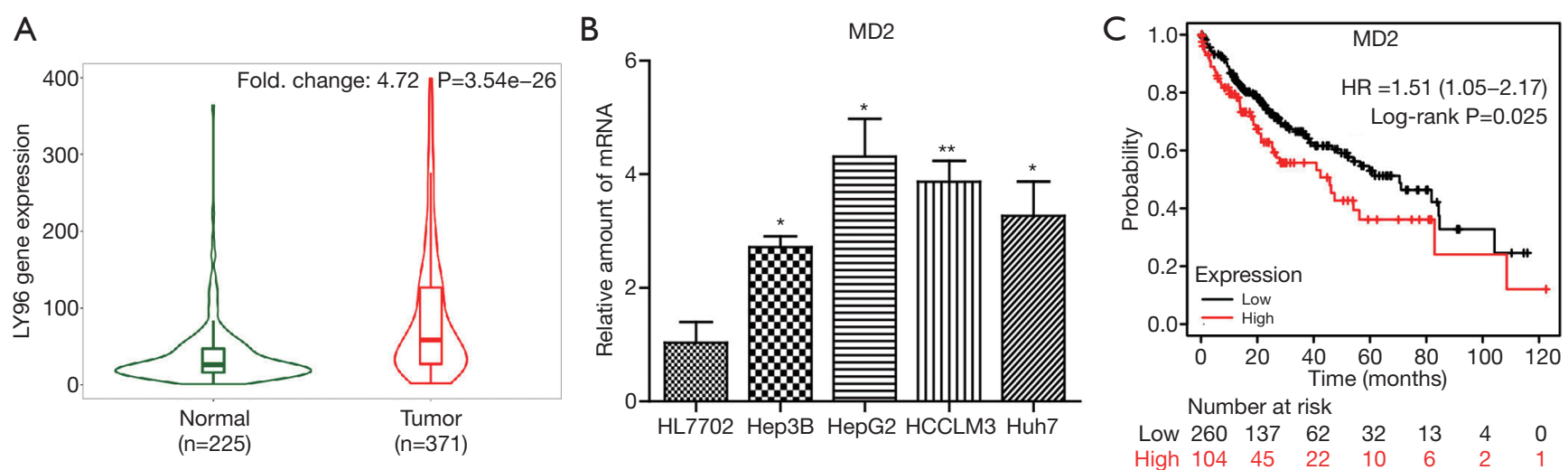

Figure $1 \mathrm{MD} 2$ was highly expressed in hepatoma carcinoma and was associated with prognosis. (A) The TNMplot database was used to analyze the MD2 levels in HCC and normal liver. (B) qRT-PCR analysis of MD2 mRNA levels in HL7702, Hep3B, HepG2, HCCLM3, and Huh7. (C) The Human Protein Atlas database was used to analyze the relationship between MD2 expression and prognosis. *, $\mathrm{P}<0.05$ compared with HL7702 group; ${ }^{* *}, \mathrm{P}<0.01$ compared with HL7702 group. MD2, myeloid differentiation protein 2; HCC, hepatocellular carcinoma; qRT-PCR, quantitative real-time PCR.

higher MD2 expression (Figure 1C). These results indicated that MD2 was highly expressed in hepatoma carcinoma and was associated with poor prognosis.

\section{Silencing of MD2 inbibited migration and invasion of HCC cells}

To explore the role and function of MD2 in HCC, we examined migration and invasion, which are important causes of tumor recurrence and metastasis, by silencing MD2 (Figure 2). First, we inhibited the expression of MD2 in HepG2 and HCCLM3 using three siRNAs, and investigated its efficiency to select the best one (Figure $2 A, 2 B, 2 E, 2 F$ ). Wound healing and transwell assays showed that MD2 silencing significantly inhibited cell migration and invasion of HepG2 cells (Figure 2C,2D) and HCCLM3 cells (Figure 2G,2H). These results indicated that silencing of MD2 prevented the progress of HCC by decreasing the cell migration and invasion ability.

\section{Silencing of MD2 slightly inbibited the proliferation of HCC cells}

We investigated the effect of MD2 on cell proliferation of HCC cells (Figure 3). CCK8 assay of HepG2 and HCCLM3 cells indicated that silencing of MD2 had no significant inhibitory effect on cell viability at $24 \mathrm{~h}$ and a slight inhibitory effect at 48 or $72 \mathrm{~h}$ (Figure 3A,3C). Colony formation assay showed that silencing of MD2 had little inhibitory effect on the number and size of cell colonies (Figure 3B,3D). These results indicated that MD2 silencing had little inhibitory effect on HCC cell proliferation.

\section{Silencing of MD2 inbibited epithelial mesenchymal transformation (EMT) of HCC cells}

EMT is an important process in tumor cell metastasis and invasion. To address the connection between MD2 and EMT, we detected the expression levels of EMT markers. As expected, silencing of MD2 prevented the procession of EMT by increasing the protein and mRNA levels of E-cadherin and Occludin, but decreasing the levels of Vimentin, $N$-cadherin, and Snail in HepG2 cells (Figure 4A,4B). Similarly, MD2 silencing in HCCLM3 cells also increased the protein and mRNA levels expression of E-cadherin and Occludin, and decreased the levels of Vimentin, N-cadherin, and Snail (Figure 4C,4D). These results showed that MD2 silencing had a significant inhibitory effect on EMT in HCC cells.

\section{Silencing of MD2 inbibited the activation of EGFR signaling}

EGFR signaling plays a key role in regulating EMT transformation of tumor cells and promoting tumor metastasis. To investigate the influence of MD2 on the EGFR signaling pathway, we detected the phosphorylation changes of EGFR and its downstream molecules, Src and 

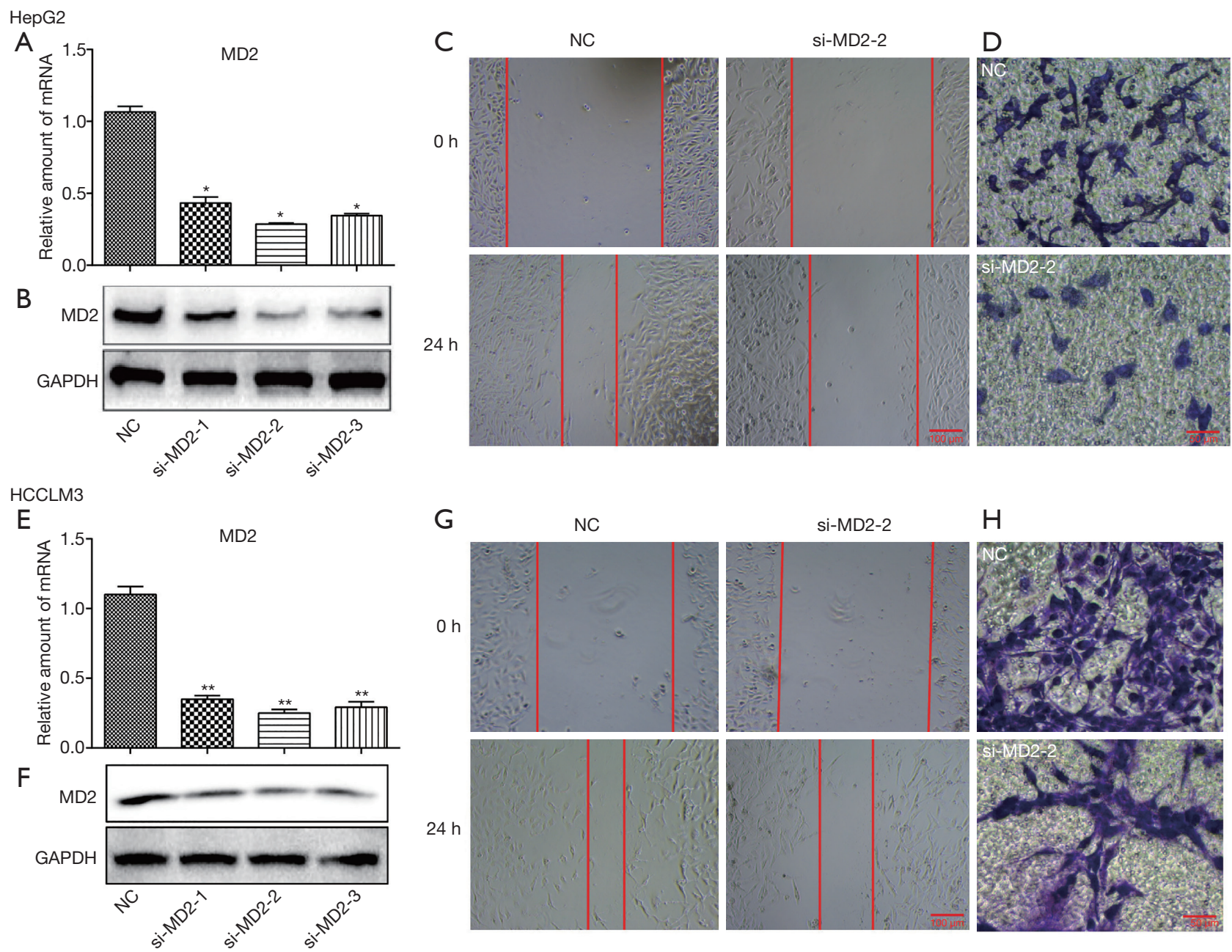

Figure 2 Silencing of MD2 inhibited cell migration and invasion. (A) qRT-PCR was used to detect the silencing efficiency of siRNA in HepG2 cells. (B) Western blot was used to detect the silencing efficiency of siRNA in HepG2 cells. (C) Wound healing assay was used for migration ability detection in HepG2 cells (100x). (D) Transwell assay and crystal violet staining were used for invasion abilities detection in HepG2 cells $(200 \times)$. (E) qRT-PCR was used to detect the silencing efficiency of siRNA in HCCLM3 cells. (F) Western blot was used to detect the silencing efficiency of siRNA in HCCLM3 cells. (G) Wound healing assay was used for migration ability detection in HCCLM3 cells $(100 \times)$. (H) Transwell assay and crystal violet staining were used for invasion abilities detection in HCCLM3 cells $(200 \times)$. *, P<0.05 compared with NC group; ${ }^{* *}, \mathrm{P}<0.05$ compared with NC group. MD2, myeloid differentiation protein 2; qRT-PCR, quantitative real-time PCR; NC, normal control.

AKT. The results demonstrated that the phosphorylation levels of EGFR, Src, and AKT were decreased in MD2silenced HepG2 and HCCLM3 cells (Figure 5A,5B). We then also inspected the changes of NF- $\mathrm{\kappa B}$ that were reported as downstream of both EGFR and MD2 signals. In the same way, MD2 silencing inhibited the phosphorylation of IкB- $\alpha$ and 065 in HepG2 and HCCLM3 cells (Figure 5A,5B). These results showed that silencing of MD2 significantly inhibited the activation of EGFR signaling in HCC cells.

\section{Discussion}

HCC is one of the main causes of cancer-related mortality worldwide, and exhibits characteristics of rapid growth and high invasiveness $(1,22)$. Therefore, it is important to investigate the mechanisms involved in HCC invasion and migration as well as explore novel therapeutic targets. Our study documented, for the first time, the effects and mechanisms of MD2 on the invasion and migration of 

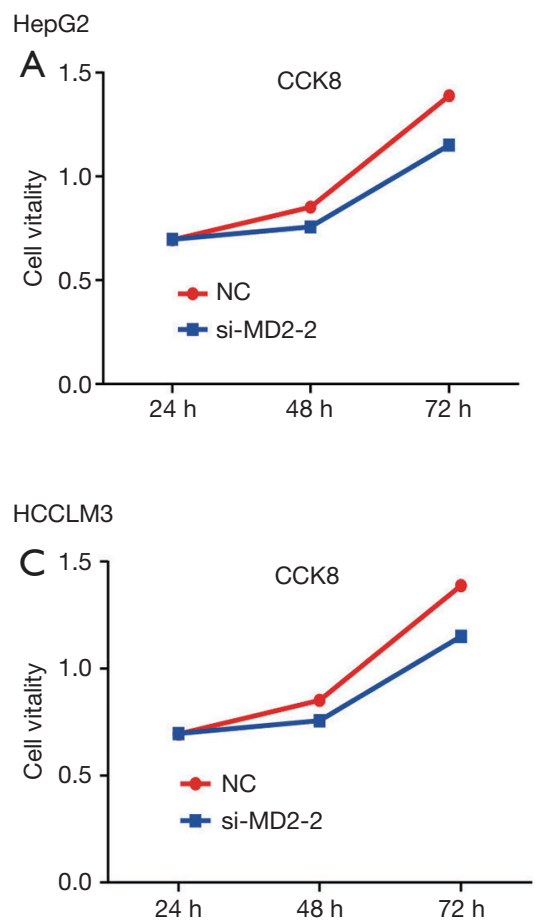

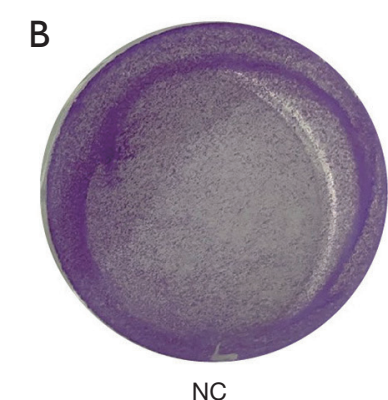

NC

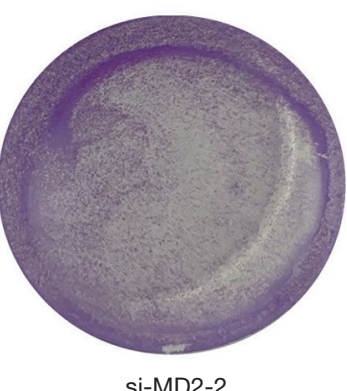

si-MD2-2
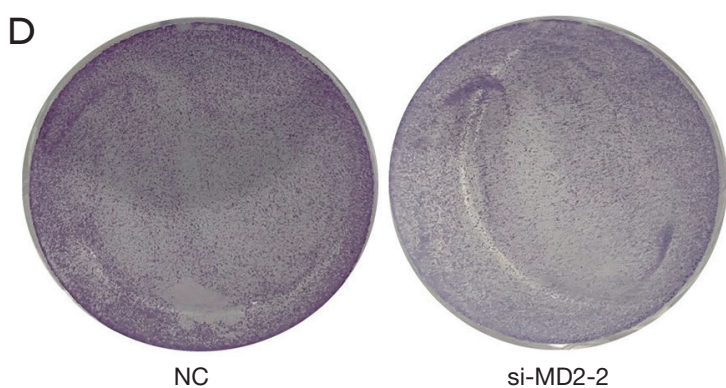

Figure 3 Silencing of MD2 slightly inhibited the proliferation of hepatoma carcinoma cells. (A) CCK8 was used to detect the cell vitality of HepG2 cells. (B) Colony formation assay and crystal violet staining were used to detect the cell proliferation of HepG2 cells. (C) CCK8 was used to detect the cell vitality of HCCLM3 cells. (D) Colony formation assay and crystal violet staining were used to detect the cell proliferation of HCCLM3 cells. MD2, myeloid differentiation protein 2; CCK8, cell counting kit 8.

\section{HCC via regulation of the EGFR pathway.}

Previous studies have found that the MD2 and TLR4 signaling pathways play an important role in acute inflammation $(23,24)$. Our previous research also identified a crucial role of these pathways in some chronic diseases, such as diabetes $(25,26)$, obesity $(27,28)$, etc. Studies in recent years have highlighted the critical function of TLR4 signaling in cancer progression, invasion, migration, angiogenesis, and immune escape $(12,29,30)$. The MD2/ TLR4 complex plays a carcinogenic effect by activating the TIRAP, PI3K/AKT, IKK- $\alpha$, and NF- $\mathrm{KB}$ signals $(31,32)$. The expression of TLR4 is significantly increased in a variety of cancers (33). High expression of TLR4 in tumor tissues is associated with poor survival in patients with various cancers $(34,35)$. Although previous studies have demonstrated the potential therapeutic implications of inhibiting TLR4 pathway in cancer treatment, few studies have focused on the specific contribution of MD2, which has been known as an essential cofactor in TLR4 signaling for many years. As far as we know, the function of MD2 on liver cancer has never been studied.
In this study, we explored the expression levels of MD2 using the TNMplot database, and found that the expression level of MD2 was higher in various tumor tissues as compared with paired normal tissues, especially in HCC. In addition, MD2 expression was upregulated in HCC cell lines, such as Hep3B, HepG2, HCCLM3, and Huh7, as compared with the human normal hepatocyte, HL7702. Survival analysis using The Human Protein Atlas database showed that higher expression of MD2 predicted poorer survival compared to lower expression. Furthermore, MD2 silencing had a weak inhibitory effect on the proliferation of HCC cells, however exhibited an obvious inhibitory effect on cell migration and invasion. Therefore, the first finding of this study was the validation of the effect of MD2 on suppressing the invasion and migration of HCC cells.

We also demonstrated that MD2 inhibited the invasion and migration ability of HCC cells via regulation of the EMT process. EMT, which is characterized by the loss of apical basal cell polarity of epithelial and the mesenchymal cells, is the key link of tumor invasion, migration, and metastasis (36-38). Inhibition of EMT regulators is an important 

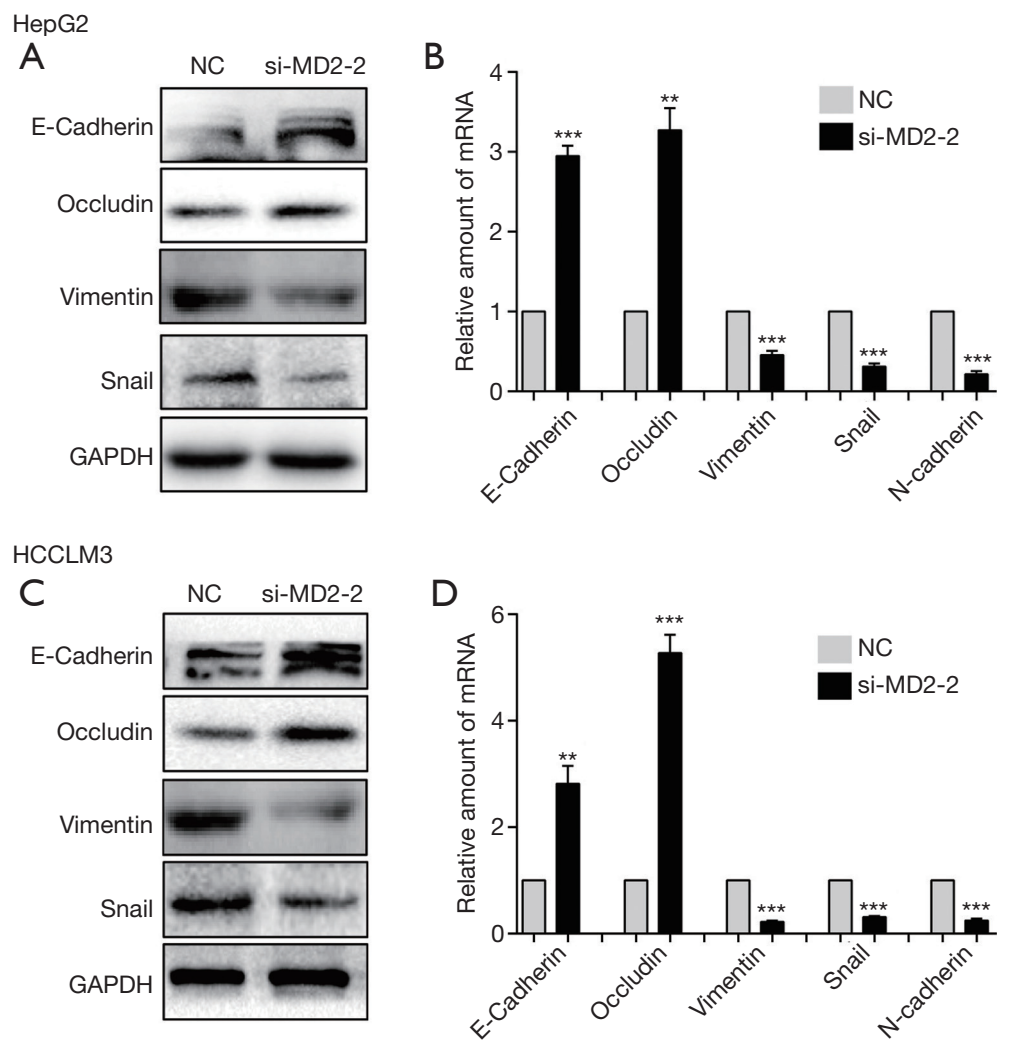

Figure 4 Silencing of MD2 inhibited cell EMT. (A) Western blot detected the expression of EMT markers in HepG2 cells. (B) qRT-PCR detected the expression of EMT markers in HepG2 cells. (C) Western blot detected the expression of EMT markers in HCCLM3 cells. (D) qRT-PCR detected the expression of EMT markers in HCCLM3 cells. **, $\mathrm{P}<0.01$ compared with NC group; ***, $\mathrm{P}<0.001$ compared with NC group. MD2, myeloid differentiation protein 2; EMT, epithelial mesenchymal transformation; qRT-PCR, quantitative real-time PCR; NC, normal control.
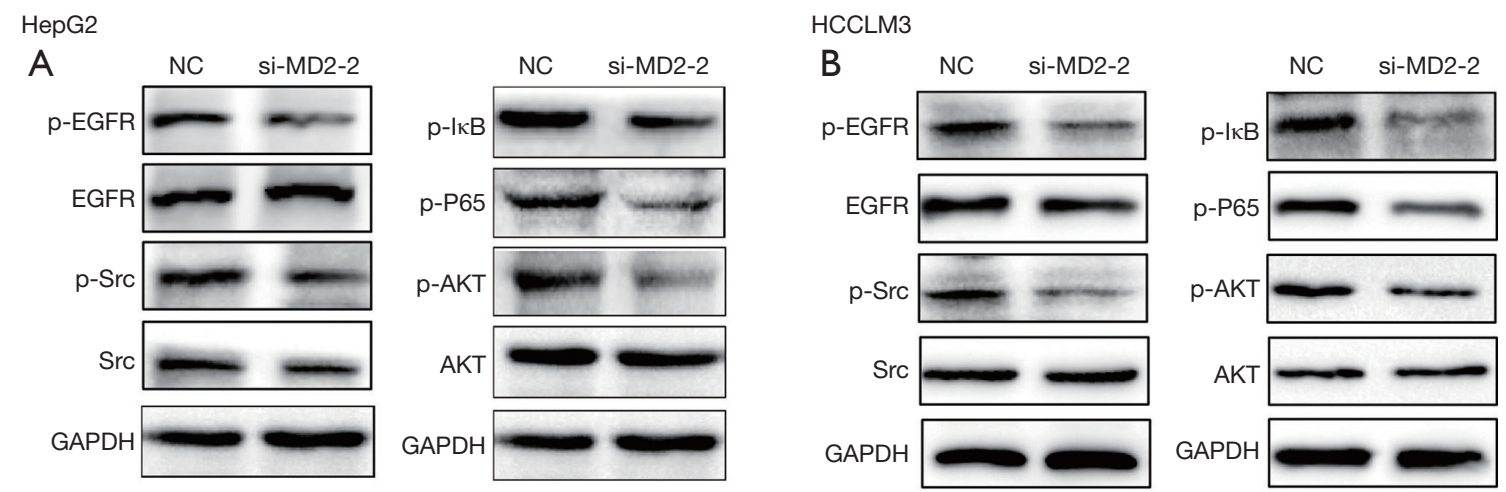

Figure 5 Silencing MD2 inhibited the activation of EGFR signaling. (A) Western blot detected the phosphorylation changes of EGFR, Src, AKT, I- $\kappa \mathrm{B} \alpha$, and p65 in HepG2 cells. (B) Western blot detected the phosphorylation changes of EGFR, Src, AKT, I- $\kappa$ B $\alpha$, and p65 in HCCLM3 cells. MD2, myeloid differentiation protein 2; EGFR, epidermal growth factor receptor; NC, normal control. 
approach in anticancer research. The multi-targeted tyrosine kinase inhibitors like sorafenib and regorafenib have an established place in the systemic treatment for advanced HCC. It was reported that sorafenib (39) and regorafenib (40) could suppress the EMT of HCC cells. The expression levels of various molecules also change during EMT. The expression of epithelial molecular markers E-cadherin and the occlusal protein (Occludin) were downregulated, while the expression of the E-cadherin transcription factor (Snail) was upregulated in the EMT process, which simultaneously acquired mesenchymal markers such as Vimentin and $\mathrm{N}$-cadherin $(41,42)$. E-cadherin mainly mediates cell-to-cell adhesion and participates widely in cell-to-cell connection, and is crucial for maintaining the integrity of normal epithelial cells. Snail can recognize and bind the promoter sequence of the E-cadherin gene, inhibit the transcription of E-cadherin, affect the adhesion between epithelial cells, and promote the occurrence of EMT (43). Occludin, one of the tight junction proteins between cells, can increase the transmembrane resistance of epithelial cells and prevent cell diffusion (44). Vimentin is abundantly expressed in numerous tumor types and is known as a canonical marker of EMT reprogramming, which is related to the acquisition of invasive and migratory tumor cell phenotypes (45). Serving as an indicator of ongoing EMT, N-cadherin is expressed in almost all interstitial cells and is associated with the development of various cancer types (46). As expected, the silencing of MD2 could block the EMT process of hepatoma cells, accompanied by the upregulation of E-cadherin and Occludin expression, and the downregulation of Snail, Vimentin, and $\mathrm{N}$-cadherin expression.

As the expression product of the proto-oncogene CerbB-1, EGFR plays an important role in a variety of cancer processes, such as regulating the cell cycle, inducing angiogenesis, accelerating tumor invasion and metastasis, promoting tumor drug resistance, and so on (47). Also, studies have indicated that the EGFR signaling pathway is closely related to the occurrence of EMT in lung, ovarian, uterine, liver, and other cancers. EGFR can mediate the occurrence of tumor EMT through Src, AKT, TGF- $\beta$, NF$\kappa \mathrm{B}, \mathrm{MAPK}$, and other signals $(48,49)$. Src, NF- $\mathrm{kB}$, and AKT are vital downstream molecules in the EGFR signaling pathway. Studies have demonstrated that Src inhibitors can significantly inhibit the EMT process of tumor cells, while Src activation can destroy cadherin-dependent intercellular adhesion and promote the interstitial phenotype of tumor cells (50). Increased expression and activity of NF- $\kappa \mathrm{B}$ leads to the loss of E-cadherin through direct regulation of the target gene of Snail (51). Moreover, the activation of NF$\mathrm{\kappa B}$ can upregulate the expression of Vimentin, thereby indirectly affecting the level of Occludin (52). AKT signal activation can directly upregulate the expression of EMTrelated transcription factors such as Snail, Slug, Twist, and ZEB, as well as inhibit the expression and promote the degradation of intracellular E-cadherin $(53,54)$. In this study, we investigated these EMT-related signaling pathways to clarify the regulatory mechanism of MD2. We found that MD2 silencing could inhibit the activation of the EGFR signaling pathway via suppression of the phosphorylation of EGFR and its downstream molecules Src, NF- $\kappa \mathrm{B}$, and AKT.

\section{Conclusions}

In summary, our data indicated that silencing of MD2 could block the occurrence of EMT by inhibiting the EGFR signaling pathway, thereby inhibiting the migration and invasion of HCC cells. These findings reveal that MD2 blockage may be a novel method in regulating the invasion and migration of HCC.

\section{Acknowledgments}

Funding: This research was funded by the National Natural Science Foundation of China (Grant No. 81903898, 81773819,81973396 , and 82003851), the Zhejiang Provincial Natural Science Foundation of China (Grant No. YY19H310003), and the Medical Science and Technology Program of Zhejiang province (Grant No. 2013KYA027, 2017190933, 2016KYB038, 2019ZD024, and 2019KY037).

\section{Footnote}

Reporting Checklist: The authors have completed the MDAR reporting checklist. Available at https://dx.doi. org/10.21037/jgo-21-362

Data Sharing Statement: Available at https://dx.doi. org/10.21037/jgo-21-362

Conflicts of Interest: All authors have completed the ICMJE uniform disclosure form (available at https://dx.doi. org/10.21037/jgo-21-362). All authors reported that this manuscript was funded by the National Natural Science Foundation of China (Grant No. 81903898, 81773819, 81973396, and 82003851), the Zhejiang Provincial Natural 
Science Foundation of China (Grant No. YY19H310003), and the Medical Science and Technology Program of Zhejiang province (Grant No. 2013KYA027, 2017190933, 2016KYB038, 2019ZD024, and 2019KY037). The authors have no other conflicts of interest to declare.

Ethical Statement: The authors are accountable for all aspects of the work in ensuring that questions related to the accuracy or integrity of any part of the work are appropriately investigated and resolved. The study was conducted in accordance with the Declaration of Helsinki (as revised in 2013). Institutional ethical approval and informed consent were waived.

Open Access Statement: This is an Open Access article distributed in accordance with the Creative Commons Attribution-NonCommercial-NoDerivs 4.0 International License (CC BY-NC-ND 4.0), which permits the noncommercial replication and distribution of the article with the strict proviso that no changes or edits are made and the original work is properly cited (including links to both the formal publication through the relevant DOI and the license). See: https://creativecommons.org/licenses/by-nc-nd/4.0/.

\section{References}

1. Fitzmaurice C, Abate D, Abbasi N, et al. Global, regional, and national cancer incidence, mortality, years of life lost, years lived with disability, and disability-adjusted life-years for 29 cancer groups, 1990 to 2017: a systematic analysis for the global burden of disease study. JAMA Oncol 2019;5:1749-68.

2. Pang RW, Poon RT. From molecular biology to targeted therapies for hepatocellular carcinoma: the future is now. Oncology 2007;72 Suppl 1:30-44.

3. Castroagudín JF, Delgado M, Villanueva A, et al. Safety of percutaneous ethanol injection as neoadjuvant therapy for hepatocellular carcinoma in waiting list liver transplant candidates. Transplant Proc 2005;37:3871-3.

4. Bray F, Ferlay J, Soerjomataram I, et al. Global cancer statistics 2018: GLOBOCAN estimates of incidence and mortality worldwide for 36 cancers in 185 countries. CA Cancer J Clin 2018;68:394-424.

5. Siegel RL, Miller KD, Jemal A. Cancer statistics, 2019. CA Cancer J Clin 2019;69:7-34.

6. Shimazu R, Akashi S, Ogata H, et al. MD-2, a molecule that confers lipopolysaccharide responsiveness on Toll-like receptor 4. J Exp Med 1999;189:1777-82.
7. Ohto U, Fukase K, Miyake K, et al. Crystal structures of human MD-2 and its complex with antiendotoxic lipid IVa. Science 2007;316:1632-4.

8. Kim HM, Park BS, Kim JI, et al. Crystal structure of the TLR4-MD-2 complex with bound endotoxin antagonist Eritoran. Cell 2007;130:906-17.

9. Chen G, Xiao B, Chen L, et al. Discovery of new MD2targeted anti-inflammatory compounds for the treatment of sepsis and acute lung injury. Eur J Med Chem 2017;139:726-40.

10. Robison A, Snyder DT, Christensen K, et al. Expression of human TLR4/myeloid differentiation factor 2 directs an early innate immune response associated with modest increases in bacterial burden during Coxiella burnetii infection. Innate Immun 2019;25:401-11.

11. Zhan Z, Xie X, Cao H, et al. Autophagy facilitates TLR4and TLR3-triggered migration and invasion of lung cancer cells through the promotion of TRAF6 ubiquitination. Autophagy 2014;10:257-68.

12. Liu WT, Jing YY, Yu GF, et al. Toll like receptor 4 facilitates invasion and migration as a cancer stem cell marker in hepatocellular carcinoma. Cancer Lett 2015;358:136-43.

13. Li R, Zhou R, Wang H, et al. Gut microbiota-stimulated cathepsin K secretion mediates TLR4-dependent M2 macrophage polarization and promotes tumor metastasis in colorectal cancer. Cell Death Differ 2019;26:2447-63.

14. Tang $X, Z$ hu $Y$. TLR4 signaling promotes immune escape of human colon cancer cells by inducing immunosuppressive cytokines and apoptosis resistance. Oncol Res 2012;20:15-24.

15. Haricharan S, Brown P. TLR4 has a TP53-dependent dual role in regulating breast cancer cell growth. Proc Natl Acad Sci U S A 2015;112:E3216-25.

16. Jain S, Suklabaidya S, Das B, et al. TLR4 activation by lipopolysaccharide confers survival advantage to growth factor deprived prostate cancer cells. Prostate 2015;75:1020-33.

17. Dickinson SE, Wondrak GT. TLR4-directed molecular strategies targeting skin photodamage and carcinogenesis. Curr Med Chem 2018;25:5487-502.

18. Szajnik M, Szczepanski MJ, Czystowska M, et al. TLR4 signaling induced by lipopolysaccharide or paclitaxel regulates tumor survival and chemoresistance in ovarian cancer. Oncogene 2009;28:4353-63.

19. Jiang N, Xie F, Chen L, et al. The effect of TLR4 on the growth and local inflammatory microenvironment of HPV-related cervical cancer in vivo. Infect Agent Cancer 
2020;15:12.

20. Kohtz PD, Halpern AL, Eldeiry MA, et al. Toll-like receptor-4 is a mediator of proliferation in esophageal adenocarcinoma. Ann Thorac Surg 2019;107:233-41.

21. Chen CL, Tsukamoto H, Liu JC, et al. Reciprocal regulation by TLR4 and TGF- in tumor-initiating stemlike cells. J Clin Invest 2013;123:2832-49.

22. Villanueva A. Hepatocellular Carcinoma. N Engl J Med 2019;380:1450-62.

23. Chen L, Fu W, Zheng L, et al. Recent progress in the discovery of myeloid differentiation 2 (MD2) modulators for inflammatory diseases. Drug Discov Today 2018;23:1187-202.

24. Tandon A, Harioudh MK, Ishrat N, et al. An MD2derived peptide promotes LPS aggregation, facilitates its internalization in THP-1 cells, and inhibits LPSinduced pro-inflammatory responses. Cell Mol Life Sci 2018;75:2431-46.

25. Wang Y, Fang Q, Jin Y, et al. Blockade of myeloid differentiation 2 attenuates diabetic nephropathy by reducing activation of the renin-angiotensin system in mouse kidneys. Br J Pharmacol 2019;176:2642-57.

26. Wang Y, Luo W, Han J, et al. MD2 activation by direct AGE interaction drives inflammatory diabetic cardiomyopathy. Nat Commun 2020;11:2148.

27. Fang Q, Wang L, Yang D, et al. Blockade of myeloid differentiation protein 2 prevents obesity-induced inflammation and nephropathy. J Cell Mol Med 2017;21:3776-86.

28. Fang Q, Wang J, Zhang Y, et al. Inhibition of myeloid differentiation factor-2 attenuates obesity-induced cardiomyopathy and fibrosis. Biochim Biophys Acta Mol Basis Dis 2018;1864:252-62.

29. Fu XQ, Liu B, Wang YP, et al. Activation of STAT3 is a key event in TLR4 signaling-mediated melanoma progression. Cell Death Dis 2020;11:246.

30. Sun Z, Luo Q, Ye D, et al. Role of toll-like receptor 4 on the immune escape of human oral squamous cell carcinoma and resistance of cisplatin-induced apoptosis. Mol Cancer 2012;11:33.

31. Sun Y, Wu C, Ma J, et al. Toll-like receptor 4 promotes angiogenesis in pancreatic cancer via PI3K/AKT signaling. Exp Cell Res 2016;347:274-82.

32. Mai CW, Kang YB, Pichika MR. Should a Toll-like receptor 4 (TLR-4) agonist or antagonist be designed to treat cancer? TLR-4: its expression and effects in the ten most common cancers. Onco Targets Ther 2013;6:1573-87.

33. Huang B, Zhao J, Li H, et al. Toll-like receptors on tumor cells facilitate evasion of immune surveillance. Cancer Res 2005;65:5009-14. Erratum in: Cancer Res 2005;65:9108. Erratum in: Cancer Res 2019;79:4305.

34. Wang Q, Zhang X, Xiao T, et al. Prognostic role of Tolllike receptors in cancer: a meta-analysis. Ther Clin Risk Manag 2018;14:1323-30.

35. Zahran AM, Zahran ZAM, El-Badawy O, et al. Prognostic impact of toll-like receptors 2 and 4 expression on monocytes in Egyptian patients with hepatocellular carcinoma. Immunol Res 2019;67:157-65.

36. Sánchez-Tilló E, Liu Y, de Barrios O, et al. EMT-activating transcription factors in cancer: beyond EMT and tumor invasiveness. Cell Mol Life Sci 2012;69:3429-56.

37. Kalluri R, Weinberg RA. The basics of epithelialmesenchymal transition. J Clin Invest 2009;119:1420-8.

38. Abderrazak A, Couchie D, Mahmood DF, et al. Antiinflammatory and antiatherogenic effects of the NLRP3 inflammasome inhibitor arglabin in ApoE2.Ki mice fed a high-fat diet. Circulation 2015;131:1061-70.

39. Dong S, Kong J, Kong F, et al. Sorafenib suppresses the epithelial-mesenchymal transition of hepatocellular carcinoma cells after insufficient radiofrequency ablation. BMC Cancer 2015;15:939.

40. Zhang N, Zhang S, Wu W, et al. Regorafenib inhibits migration, invasion, and vasculogenic mimicry of hepatocellular carcinoma via targeting ID1-mediated EMT. Mol Carcinog 2021;60:151-63.

41. Ramesh V, Brabletz T, Ceppi P. Targeting EMT in cancer with repurposed metabolic inhibitors. Trends Cancer 2020;6:942-50.

42. Davis FM, Stewart TA, Thompson EW, et al. Targeting EMT in cancer: opportunities for pharmacological intervention. Trends Pharmacol Sci 2014;35:479-88.

43. Wang Y, Shi J, Chai K, et al. The role of snail in EMT and tumorigenesis. Curr Cancer Drug Targets 2013;13:963-72.

44. Cummins PM. Occludin: one protein, many forms. Mol Cell Biol 2012;32:242-50.

45. Strouhalova K, P echová M, Gandalovi ová A, et al. Vimentin intermediate filaments as potential target for cancer treatment. Cancers (Basel) 2020;12:184.

46. Loh CY, Chai JY, Tang TF, et al. The E-cadherin and $\mathrm{N}$-cadherin switch in epithelial-to-mesenchymal transition: signaling, therapeutic implications, and challenges. Cells 2019;8:1118.

47. Sigismund S, Avanzato D, Lanzetti L. Emerging functions of the EGFR in cancer. Mol Oncol 2018;12:3-20.

48. Tulchinsky E, Demidov O, Kriajevska M, et al. EMT: A mechanism for escape from EGFR-targeted therapy 
in lung cancer. Biochim Biophys Acta Rev Cancer 2019;1871:29-39.

49. Lindsey S, Langhans SA. Epidermal growth factor signaling in transformed cells. Int Rev Cell Mol Biol 2015;314:1-41.

50. Guarino M. Src signaling in cancer invasion. J Cell Physiol 2010;223:14-26.

51. Hu Z, Liu X, Tang Z, et al. Possible regulatory role of Snail in NF-kB-mediated changes in E-cadherin in gastric cancer. Oncol Rep 2013;29:993-1000.

52. Xu CY, Qin MB, Tan L, et al. NIBP impacts on the expression of E-cadherin, CD44 and vimentin in

Cite this article as: Qi Y, Fang Q, Li Q, Ding H, Shu Q, Hu Y, Xin W, Fang L. MD2 blockage prevents the migration and invasion of hepatocellular carcinoma cells via inhibition of the EGFR signaling pathway. J Gastrointest Oncol 2021;12(4):18731883. doi: 10.21037/jgo-21-362 colon cancer via the NF- $\mathrm{kB}$ pathway. Mol Med Rep 2016;13:5379-85.

53. Xu W, Yang $Z, \mathrm{Lu} N$. A new role for the PI3K/Akt signaling pathway in the epithelial-mesenchymal transition. Cell Adh Migr 2015;9:317-24.

54. Rafael D, Doktorovová S, Florindo HF, et al. EMT blockage strategies: targeting Akt dependent mechanisms for breast cancer metastatic behaviour modulation. Curr Gene Ther 2015;15:300-12.

(English Language Editor: A. Kassem) 